\title{
MATRIX FORMULATION FOR INFINITE-RANK OPERATORS
}

\author{
BALMOHAN V. LIMAYE
}

Abstract. Every finite-rank operator on a linear space $X$ is the composition of an operator from $X$ to a finite dimensional Euclidean space and of an operator from that Euclidean space to $X$. We consider operators which are the sum of a finite-rank operator and another infinite-rank operator which satisfies an invariance condition with respect to one of the two 'components' of the finiterank operator. A canonical procedure is given to reduce operator equations, eigenvalue problems and spectral subspace problems involving such operators to corresponding problems for finite matrices.

Mathematics subject classification (2010): 47A58, 47A25, 47A75.

formulation.

Keywords and phrases: finite-rank operator, operator equation, eigenspace, spectral subspace, matrix

\section{REFERENCES}

[1] M. Ahues, A. Largillier And B. V. Limaye, Spectral Computations for Bounded Operators, Chapman and Hall/CRC Press, Boca Raton, 2001.

[2] M. Ahues And B. V. Limaye, Computation of spectral subspaces for weakly singular integral operators, Numer. Funct. Anal. and Optimiz., 25 (2004), 1-14.

[3] K. E. AtKinson, The numerical solution of integral equations of the second kind, Cambridge University Press, Cambridge, 1997.

[4] L. N. Deshpande AND B. V. Limaye, On the stability of singular finite-rank methods, SIAM J. Numer. Anal., 27 (1990), 792-803.

[5] A. LARGILlier AND B. V. LimaYE, Finite-rank methods and their stability for coupled systems of operator equations, SIAM J. Numer. Anal., 33 (1996), 707-728.

[6] H. MAJidian AND E. BABOlian, An interpolation degenerate kernel method for eigenvalue problems of a class of non-compact operators, Appl. Math. Lett., 23 (2010), 885-890.

[7] R. WhitLEY, The stability of finite rank methods with applications to integral equations, SIAM J. Numer. Anal., 23 (1986), 118-134. 